Scientific Paper

\title{
Synthetic CT in assessment of anatomical and dosimetric variations in radiotherapy - procedure validation
}

\author{
Aleksandra GRZĄDZIEL ${ }^{a}$, Adam GĄDEK ${ }^{\mathrm{a}, \mathrm{b}}$, Barbara BEKMAN ${ }^{\mathrm{a}}$, Jacek WENDYKIER ${ }^{\mathrm{a}}$, Krzysztof ŚLOSAREK \\ ${ }^{a}$ Radiotherapy Planning Department, Maria Skłodowska-Curie National Research Institute of Oncology, Gliwice Branch, \\ Gliwice, Poland \\ ${ }^{b}$ Medical Physics Department, Institute of Physics, University of Silesia, Chorzów, Poland \\ *E-mail address: aleksandra.grzadziel@io.gliwice.pl
}

\begin{abstract}
Introduction: One of many procedures to control the quality of radiotherapy is daily imaging of the patient's anatomy. The CBCT (Cone Beam Computed Tomography) plays an important role in patient positioning, and dose delivery monitoring. Nowadays, CBCT is a baseline for the calculation of fraction and total dose. Thus, it provides the potential for more comprehensive monitoring of the delivered dose and adaptive radiotherapy. However, due to the poor quality and the presence of numerous artifacts, the replacement of the CBCT image with the corrected one is desired for dose calculation. The aim of the study was to validate a method for generating a synthetic CT image based on deformable image registration.

Material and methods: A Head \& Torso Freepoint phantom, model 002H9K (Computerized Imaging Reference Systems, Norfolk, USA) with inserts was imaged with CT (Computed Tomography). Then, contouring and treatment plan were created in Eclipse (Varian Medical Systems, Palo Alto, CA, USA) treatment planning system. The phantom was scanned again with the CBCT. The planning CT was registered and deformed to the CBCT, resulting in a synthetic CT in Velocity software (Varian Medical Systems, Palo Alto, CA, USA). The dose distribution was recalculated based on the created CT image.

Results: Differences in structure volumes and dose statistics calculated both on CT and synthetic CT were evaluated. Discrepancies between the original and delivered plan from 0.0 to $2.5 \%$ were obtained. Dose comparison was performed on the DVH (Dose-Volume Histogram) for all delineated inserts.

Conclusions: Our findings suggest the potential utility of deformable registration and synthetic CT for providing dose reconstruction. This study reports on the limitation of the procedure related to the limited length of the CBCT volume and deformable fusion inaccuracies.
\end{abstract}

Key words: $\mathrm{CBCT}$; synthetic $\mathrm{CT}$; deformable registration; adaptive radiotherapy.

\section{Introduction}

Conformal irradiation techniques allow for precise irradiation of well-defined patient volumes. But at the same time, it can be burdened with the risk of making geometrical errors, which may result in the deposition of too low dose in the target volume or too high dose in critical structures. Geometric errors can occur at the stage of treatment planning or irradiation session. ${ }^{1,2}$

The accuracy of therapy can be disturbed by the anatomical changes of a patient, such as weight gain or significant weight loss, involuntary movements, physiological mobility of organs, and inaccurate geometry setting by the operator. Verification of the patient position and its correction to the planned one is carried out before each therapeutic session. Daily verification of the patient position with two-dimensional (2D) and then three-dimensional (3D) images of the anatomy have become the starting point for more extensive methods of daily dose control. $^{3-7}$ The acquired verification images became the basis for calculating dose distributions delivered to the body of a patient. This can be considered as an initial step toward adaptive therapy, using daily imaging to adjust the treatment plan to the current patient anatomy. ${ }^{8-10}$

Nowadays one of the most commonly used methods for patient position verification in radiotherapy is CBCT (Cone Beam Computed Tomography). CBCT involves acquiring a series of $\mathrm{kV}$ images while the gantry is rotating. Computer software using the Feldkamp-Davis-Kress algorithm reconstructs the image after imaging. ${ }^{11-13}$ Comparing the CBCT image with the CT reference image from the treatment planning system provides a range of clinically relevant information. The calculated values of shifts and rotations are translated into a table movement in the sagittal, coronal and

(C) 2020 Aleksandra Grządziel, Adam Gądek, Barbara Bekman, Jacek Wendykier, Krzysztof Ślosarek. This is an open access article licensed under the Creative Commons Attribution-NonCommercial-NoDerivs License (http://creativecommons.org/licenses/by-nc-nd/3.0/). 
transverse planes. Thus, the corrected patient position matches the assumed one. The usage of CBCT can be extended to calculate dose distributions for the mentioned adaptive radiotherapy. In order to use any $\mathrm{CT}$ scanner for calculating the dose distribution, it is necessary to determine the relationship between the tissue density and the corresponding Hounsfield Unit (HU) values. Similarly, for CBCT scanners mounted on accelerators, such a calibration procedure in the treatment planning system is needed. Dose distribution calculations based on CBCT images without calibrating these devices is not recommended. ${ }^{14-16}$ Still, the quality of CBCT scans is often worse than CT quality and thus is not a good basis for dose calculations. $^{12,17-19}$ The quality of CBCT scan acquired during the treatment session is presented in Figure 1.

These two problems could be solved if the so-called synthetic or virtual CT image was created on the base of the CBCT image. The synthetic CT image should represent the current dimensions and anatomical information of the CBCT image and reliable density of the HU. In a word, CBCT should present the anatomy of the patient from the day of irradiation and have the quality of CT images. Additional software is required for the conversion of a distorted $\mathrm{CBCT}$ image into a useful CT image. For this purpose, the algorithms for deformation fusion must be applied. Such an algorithm can propagate the $\mathrm{HU}$ unit from the $\mathrm{CT}$ to the CBCT image. A synthetic $\mathrm{CT}$ enables the fraction and total dose distribution to be recalculated. ${ }^{15,19-21}$ The authors discuss in this work the operation and usefulness of such commercial software in dose distribution calculation.

The subject of deformation fusion is an important field often discussed in the literature. ${ }^{19,15,22-25}$ Generally, medical image fusion is a registration operation of one or more imaging modalities. The main goal of that is to improve the quality of diagnostic information. The classification of image fusion can be done according to the possible operations performed on the overlaid images: rigid - when shift and rotation are done; rigid affine - displacement, rotation, scaling and cutting; projective - shift, rotation, scaling and cutting, prospective scaling and curved or deformable - voxel deformation. ${ }^{25}$ In this work, the fusion is used to create the synthetic CT based on CBCT image. The terms of fusion or synonymous registration are used alternately.

Deformation registration is useful in many aspects of radiotherapy, such as: contouring, dose deformation, dose accumulation in re-irradiation, and adaptive planning. ${ }^{19,15,22-25}$ This allows flexible adjustment of the deformed image voxels to the reference image. A voxel is the three-dimensional equivalent of a pixel, i.e., the smallest element of a twodimensional image displayed on the screen. ${ }^{22}$

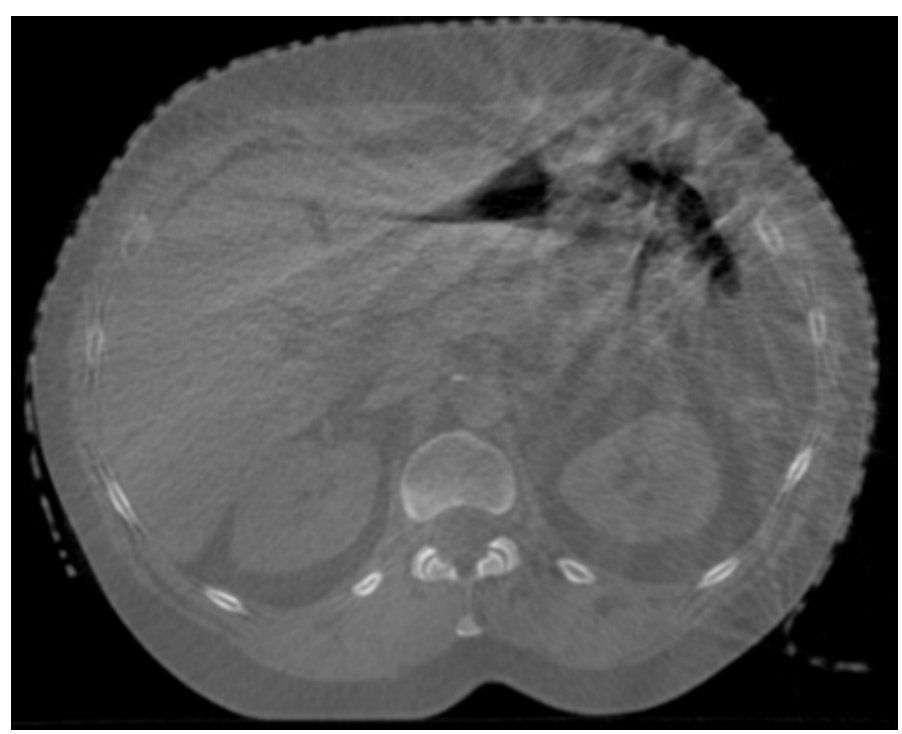

Figure 1. CBCT transversal scan of the abdomen with visible artifacts

There are many algorithms used in deformation registration, such as ANACONDA (ANAtomically CONstrained Deformation Algorithm), Morfeus or Demons, differing in the way of combining information contained in images. ${ }^{24,26-28}$ In the system validated in this study the deformation registration algorithm is used, which is a modified version of the algorithm based on the so-called B-spline curves. ${ }^{29,30}$ The curves are determined by a series of control points. An image with a coordinate system shifted to fit a reference image (fixed image) is called a moving image. The image similarity metric is determined based on the method of SSD (Sum of Squared Difference). The deformation is interpolated between the grid points. The moving image is deformed by the interpolation of the displacement vector. The displacement of interpolation points is affected only by the shift of the nearest grid points. Control points are automatically arranged in a grid overlaid on the image. The whole process is repeated in a loop until a deformed image is obtained. The process stoppage is mostly conditionally solved: either reaching the set value of the measure of image compatibility, or reaching the set number of iterations, which often gives unsatisfactory matching results.

The aim of the study is to validate the functionality of the Adapt Volume option in Velocity software (Varian Medical Systems, Palo Alto, USA), used to deform CBCT images and complement them with correct $\mathrm{HU}$ units to generate a synthetic CT image. 


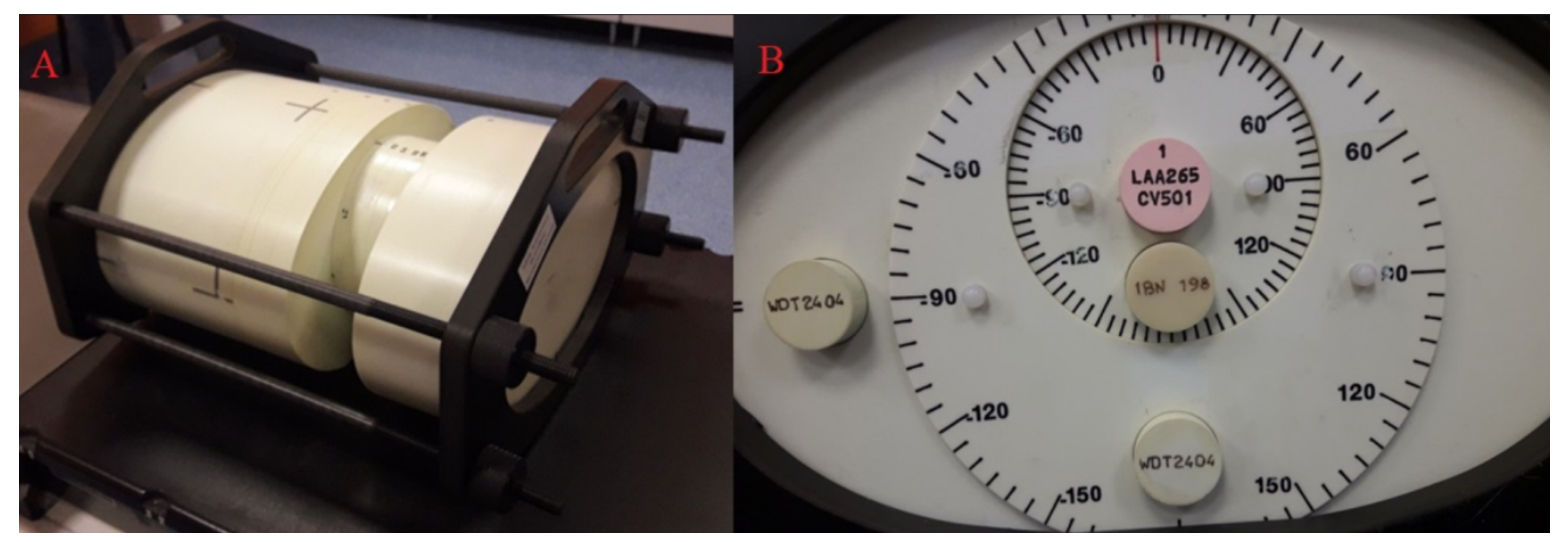

Figure 2. A - Head \& Torso Freepoint phantom, model 002H9K. B - Placement of inserts equivalent to the bone, water, and lungs

\section{Materials and methods}

This study was designed to investigate the method for creating the synthetic CT image based on a phantom, before performing it on patients. For this purpose, the Head \& Torso Freepoint phantom, model 002H9K (Computerized Imaging Reference Systems/CIRS/, Norfolk, USA) was used. Selected phantom is equipped with inserts of different densities, corresponding to the densities of human tissues. For the purpose of this study, four inserts equivalent to bones (IBN198), water (WDT2404 I, WDT2404 II), and lungs (LAA265 CV501) were used. The phantom and the way of the placement of the inserts are shown in Figure 2.

This phantom was scanned on Somatom CT (Siemens Healthineers, Erlangen, Germany) using $3 \mathrm{~mm}$ interslice distance with the protocol routinely used for radiation therapy planning. CT scans were sent to the Eclipse (Varian Medical Systems, Palo Alto, USA) planning system. Then contours of the phantom surface and inserts were introduced (Figure 3).

An initial treatment plan with 4 static $6 \mathrm{MV}$ beams was prepared. This field arrangement is frequently referred to as a box technique. Field dimensions of $20 \times 20 \mathrm{~cm}^{2}$ and equal weights were used to obtain a homogeneous dose distribution in the phantom volume. A fraction dose of $2 \mathrm{~Gy}$ and a $50 \mathrm{~Gy}$ total dose were defined. Finally, the CBCT imaging was also added in each fraction.

In the part of the experiment carried out on the accelerator the phantom was placed on the treatment table. The CBCT imaging was made in order to obtain the assumed position of the phantom. To image the patient body on the accelerator different CBCT protocols are available. Therefore, the phantom acquisition was performed according to the pelvic protocol suggested by the manufacturer with $125 \mathrm{kV}, 1080$ $\mathrm{mAs}$ and half gantry rotation. This protocol was chosen due to the dimensions and composition of the phantom. After table corrections, imaging was performed again to acquire an image of the tested object in an accurate position. This procedure aims to simulate all elements of a therapeutic session with the patient. The next step was an export of CBCT data to Eclipse

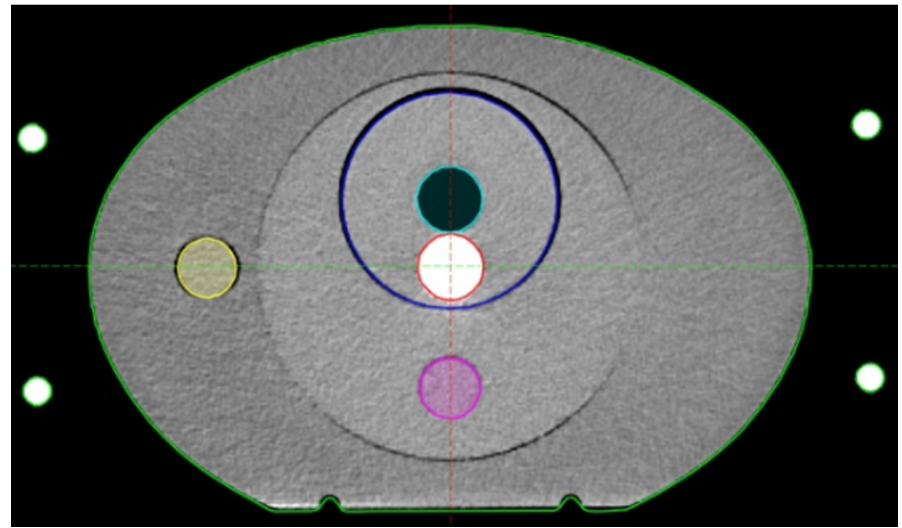

Figure 3. CT image with external contour of the phantom (green) and the inserts of water (yellow and magenta), bone (red), lung (cyan), circle insert (deep blue).

for rigid registration with the planning $\mathrm{CT}$. The reference $\mathrm{CT}$ image, the collected CBCT image and the registration file were eventually sent to the Velocity software (Varian Medical Systems, Palo Alto, USA).

Velocity is software that enables the import and integration of patient data in the DICOM format (Digital Imaging and Communications in Medicine). It contains tools to display, register and segment the volume of medical multimodal images, such as CT, MR (Magnetic Resonance), PET (Positron Emission Tomography), and SPECT (Single-Photon Emission Computed Tomography). In addition, deformation registration of two images as well as transferring the dose distribution from one image to another is practicable in the Velocity. A special tool of Adapt Volume is dedicated for generating a synthetic image based on a pair of CT and CBCT images. ${ }^{30}$ The tools utilized in this study were particularly those supporting the deformable image registration and generation of a synthetic CT image based on CBCT.

The procedure of synthetic image generation consists of several steps in Imaging and Registration modules of the Velocity system, starting from the auto alignment followed by the rigid and then deformable registration. ${ }^{30}$ 


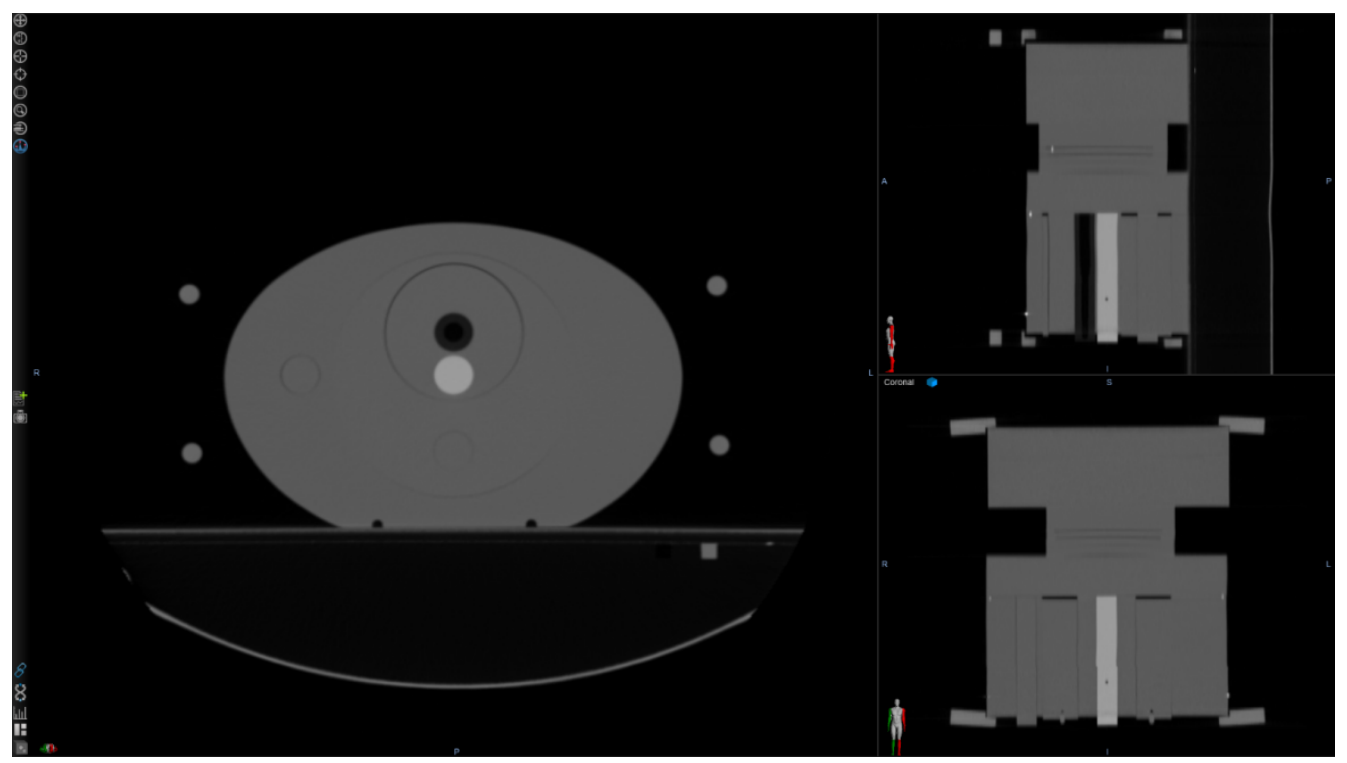

Figure 4. Synthetic CT image produced in the Velocity.

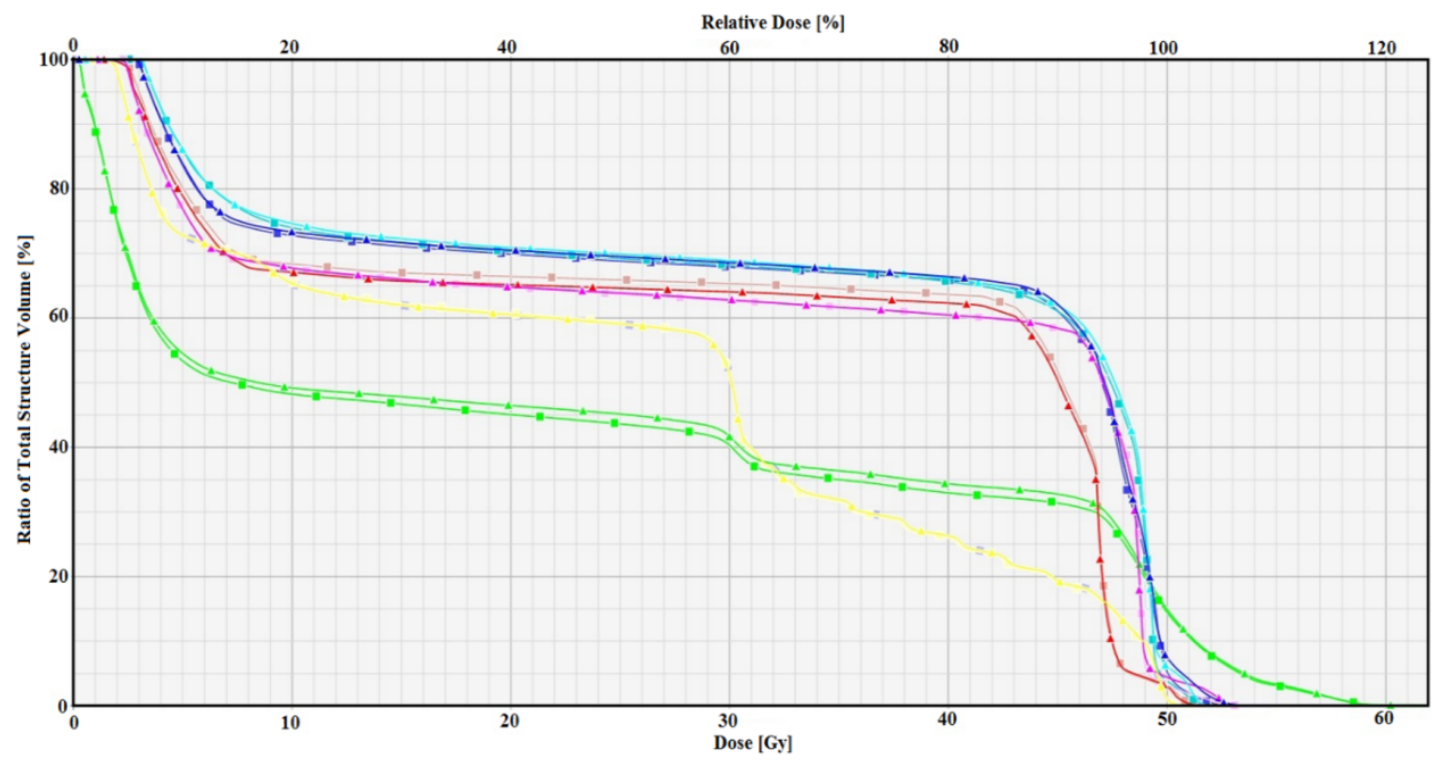

Figure 5. Dose-volume graph for original $(\Delta)$ and delivered $(\bullet)$ plans plotted in Eclipse treatment planning system. The green line represents dose distribution in phantom volume, yellow and magenta - in water insert, red - in bone insert, cyan - in lung insert, deep blue - in circle insert.

However, this action need not be described in detail here, because of the specificity of this software. Actions performed in these two modules come down to performing a deformable image fusion and replacing pixels on the CBCT image with densities from the planning CT. The generated synthetic CT image (Figure 4) was exported to the Eclipse system

The procedure validation was continued in External Beam Planning module, where the initially created treatment plan was copied into a synthetic CT with the structures and then the dose distribution was recalculated. The differences in dose distributions and structure volumes, obtained based both on planning CT and CBCT from the day of treatment, were analyzed on the basis of DVH (Dose -Volume Histogram).
Assessment of overall treatment is also possible in the Velocity system. Structures contoured for the planning can be registered to daily synthetic CT images. Hence, an adaptive and total version of the histogram can be plotted ${ }^{30}$.

\section{Results}

By generating a synthetic CT image, the comparison of dose distribution in both the original and delivered plan was feasible. The differences in doses for contoured structures were shown thanks to comparing dose statistics in planning and synthetic CT volume on DVH (Figure 5). Values of the following doses were analyzed: $D_{\min }$ (minimal dose), $D_{\max }$ (maximal dose), $\mathrm{D}_{\text {mean }}$ (mean dose), $\mathrm{D}_{\text {mod }}$ (modal dose), and $\mathrm{D}_{\text {med }}($ median dose). 
Table 1. Differences in doses and volumes of delineated structures.

\begin{tabular}{|c|c|c|c|c|c|c|}
\hline CONTOUR & Volume diff. [\%] & $\begin{array}{c}D_{\min } \\
\text { diff. }[\%]\end{array}$ & $\begin{array}{c}D_{\max } \\
\text { diff. }[\%]\end{array}$ & $\begin{array}{c}D_{\text {mean }} \\
\text { diff. }[\%]\end{array}$ & $\begin{array}{c}D_{\text {mod }} \\
\text { diff. }[\%]\end{array}$ & $\begin{array}{c}D_{\text {med }} \\
\text { diff. }[\%]\end{array}$ \\
\hline EXTERNAL & 5.6 & 0.0 & 1.0 & 1.2 & 0.2 & 2.4 \\
\hline IBN198 & 0.5 & 2.5 & 0.3 & 1.2 & 0.0 & 0.3 \\
\hline WDT2404 I & 7.3 & 1.8 & 0.5 & 0.1 & 0.0 & 0.2 \\
\hline WDT2404 II & 0.1 & 1.3 & 0.1 & 0.1 & 0.1 & 0.1 \\
\hline LAA265 & 1.8 & 0.1 & 0.0 & 0.6 & 0.0 & 0.4 \\
\hline CIRCLE & 4.2 & 0.0 & 0.2 & 0.7 & 0.3 & 0.3 \\
\hline
\end{tabular}

Dose differences from 0.0 to 2.5 percent were obtained for the phantom used in the study. The smallest differences were found between the modal doses. For the three structures no difference in the $\mathrm{D}_{\text {mod }}$ values was noted. The biggest dose differences were demonstrated for the minimum dose for one delineated structure (Table 1). Also, the differences between volumes of the structures contoured on the CT and synthetic CT were found. A value of 7.3 percent of the volume difference was demonstrated for one of the inserts.

\section{Discussion}

The study was created having in mind new software potentially useful in recalculating dose distribution on the new anatomy. If the patient volume is similar to the planned one, then the fraction dose can be delivered as wanted. However, if any alterations in anatomy occur, then changes in dose distribution are also expected. From that, there is only one step to the idea of adjusting the treatment plan to temporal patient volume, i.e., to adaptive radiotherapy. ${ }^{19,15,22-25}$ Interfraction changes in anatomy and patient setup are visible on daily CBCT images. This is a valuable imaging modality, but it also has some limitations. Numerous artifacts can appear in CBCT, thus making the planned dose distribution disturbed. Ring artifacts are one of the most common artifacts in CBCT. The conical beam scans a large area. To encompass a large volume, the detector is offset from the center of rotation. This results in an annular artifact in the axial plane. Also, beam hardening artifacts appear as dark spots or streaks around high-density materials. The limited rotation speed of X-ray source makes CBCT images more susceptible to motion artifacts due to the extended acquisition time. Movement of anatomical structures during scanning leads to streaks from high-contrast objects, such as bones or air cavities. ${ }^{12,17-19}$ These defects may disqualify older versions of devices in the reconstruction of dose distribution.

Recent studies have adopted different methods to achieve CBCT corrections, relying on deformable registration of planning CT to the CBCT or so-called SCA (scatter-correctionalgorithm) used for digitally reconstructed radiographs (DRR) of the synthetic CT. ${ }^{16,31-36}$

This study demonstrates the first approach to reduce poor CBCT image quality. We observed that the artifacts from highdensity rods located in the phantom, visible in the CBCT image i.e. dark spots and dark and white streaks, have been corrected and invisible in the synthetic CT images. In addition, the circular artifacts were also reduced. The use of tested software eliminated possible artifacts from the CBCT image. Generating synthetic CT scans causes incorrect voxels to be overwritten with valid values. Due to this, they can be considered as valuable data for treatment control.

However, this procedure also presents some difficulties for dose distribution re-planning. The first limitation of the procedure is the imperfect composite of CBCT and CT images. The length of the area scanned with the use of CBCT is limited by the conical beam breadth. Therefore, the Adapt Volume tool overrides HU values in the CBCT volume which also has limited width. Outside the overlap of CT and CBCT volumes, the voxels of CT image are copied, thus the adjacent residue is a fragment of the CT image. ${ }^{30}$ Therefore, some discontinuity of the image can be observed (Figure 6).
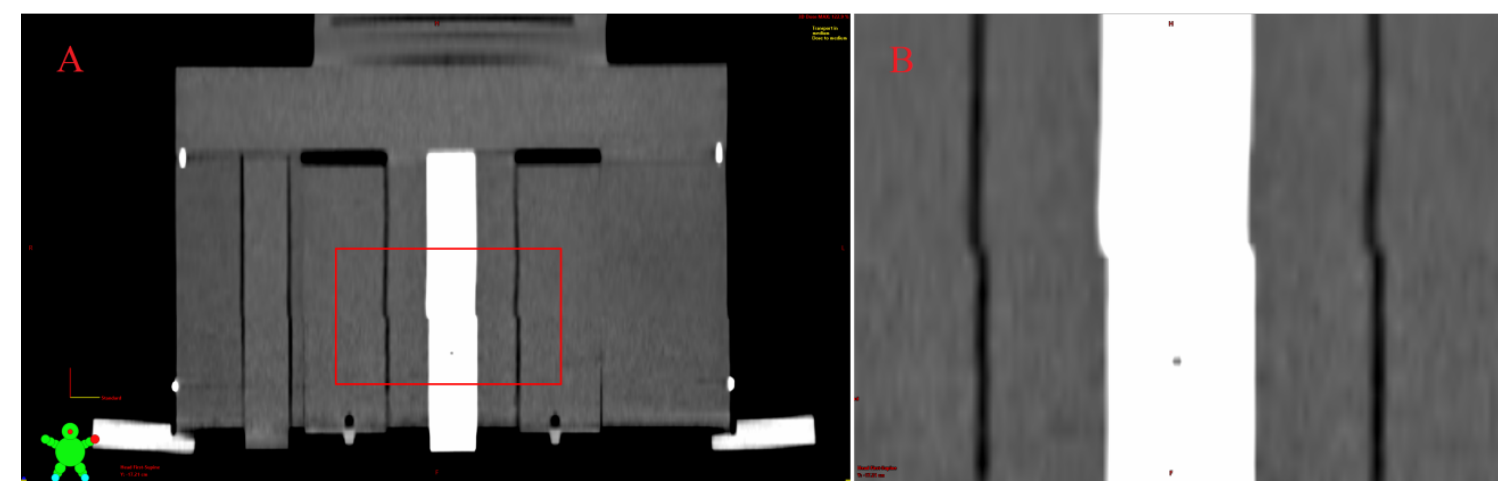

Figure 6. Breaking of the phantom image composed of CBCT and CT. A. Full view of frontal scan. B. Zoom view. 


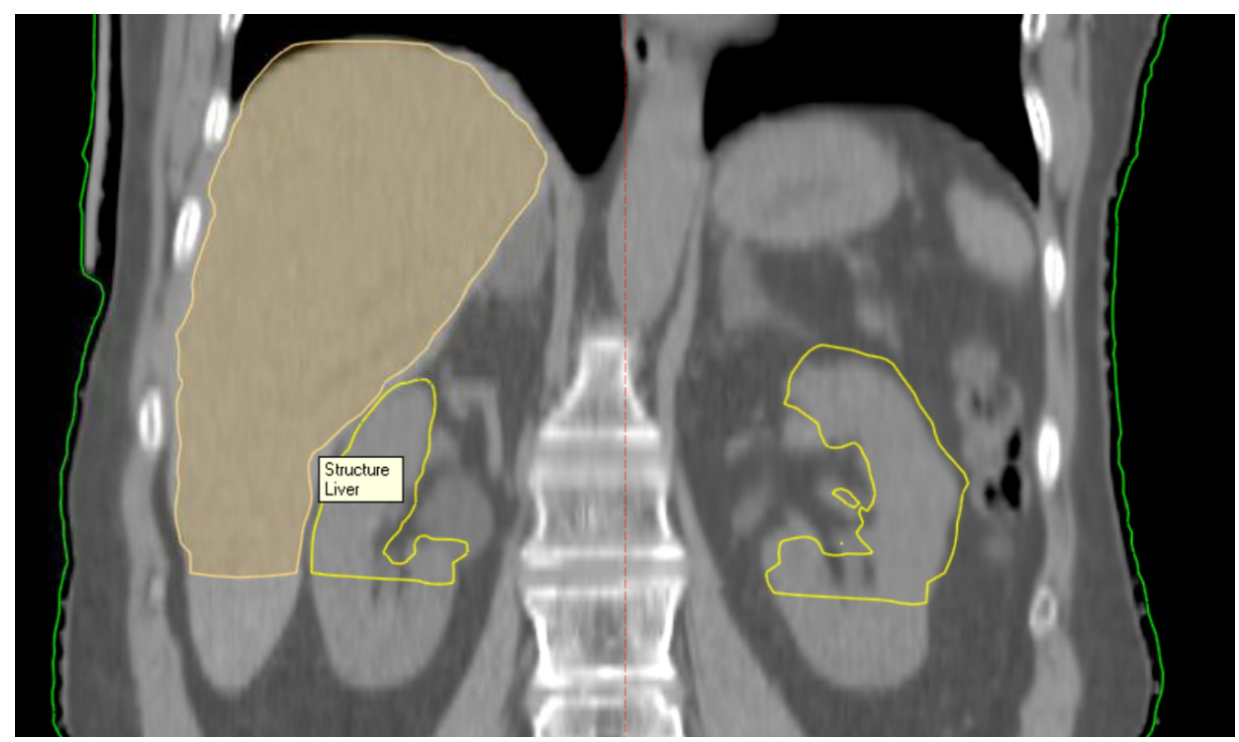

Figure 7. Synthetic CT, i.e., planning CT reshaped to CBCT and anatomy structures deformed and registered to the new volume. Visible incomplete contours of the kidneys and liver.

For this reason, uneven and discontinuous contours are produced, in particular for structures with large dimensions (Figure 7). And thus it is difficult to assess daily dose distribution and its changes in the course of the treatment. These findings suggest the potential utility of synthetic CT for providing a reasonable estimation of cumulative dose for small volume cases rather than large ones.

For quite small structures, the problem of imperfect deformation could be solved by re-contouring the structures. However, this approach also has a number of disadvantages. The new delineation is affected by human knowledge and error. Discrepancies in the volume of contours are obvious. Another disadvantage is that anatomical structure definition is mostly done based on different image modalities such as CT, MR, PET. Structures poorly visible on a synthetic CT scan can be more accurately determined on other data sets. Therefore, another multimodal image fusion would be necessary. For this reason, the deformable registration of structures seems more advantageous to the user.

A new method of generating synthetic CT examination was performed on the phantom. The differences found in dose distribution (from 0.0 to $2.5 \%$ ) were caused by a small change in the arrangement of the inserts in the phantom and subtle differences in the contouring. The resulting differences can be considered as a method error. Quite a large difference in the volume of the external structure can be caused by the automatic contouring function. The values of differences in the volumes of other structures (from 0.1 to $7.3 \%$ ) are more significant for determining the method uncertainty. The phantom with its parts is a rigid body. In particular, the circular structure is a fixed element that cannot be removed. Nonetheless, there are differences in the volumes of these structures. The obtained values can be considered as a method error. Hence, it should be remembered that the offered method is not flawless and perfect. The differences observed in patient volumes and dose distributions will be larger and hence determinable. It is commonly known that patient setup is much more demanding. Besides, various volume changes are expected in different treatment sites. In abdomen and chest anatomy any motion affects the original plan. Dose discrepancies can also occur due to tumor shrinkage or patient weight changes. All these factors are illustrated in the dose distribution recalculated on synthetic CT. A potential application of the procedure is a verification of target coverage, conformity of the dose distribution as well as dose delivered to risk structures.

Taking into account the observed disturbances in the image of moving parts of the patient body, the validation of the procedure should be extended to tests on phantom reproducing tissue motion.

\section{Conclusions}

In this study, the authors validated the algorithm that produces synthetic CT image employing deformable image registration. The suitability of the synthetic CT images was demonstrated as well. Generated synthetic CT, based on everyday CBCT volumes can solve some problem of CBCT image quality. The investigated operation performed for the phantom shows possibilities for the consideration of changes in the patient's anatomy and dose distribution. Ultimately though, the algorithm outcomes i.e. the resulting synthetic images and dose recalculations are always interpreted and evaluated by a medical physicist. Then, the newly created data set can be used as a potential volume in cumulative dose estimation, dose recalculation, and offline adaptive radiotherapy.

The work showed some limitations of the validated tool. Despite the limitations of the developed procedure, synthetic images can be utilized to analyze summary treatment plans in volumes with small sizes. 


\section{References}

1. Wyman DR, Ostapiak OZ, Gamble LM. Analysis of mechanical sources of patient alignment errors in radiation therapy. Med Phys 2002;29(11). https://doi.org/10.1118/1.1517047

2. Ezzell G, Chera B, Dicker A et al. Common error pathways seen in the RO-ILS data that demonstrate opportunities for improving treatment safety. Pract Radiat Oncol 2018;8(2):123-132

3. Goff PH, Harrison LB, Furhang E et al. 2D kV orthogonal imaging with fiducial markers is more precise for daily image guided alignments than soft-tissue cone beam computed tomography for prostate radiation therapy. Adv Radiat Oncol 2017;2(3):420-428

4. Goyal S, Kataria T, Image Guidance in Radiation Therapy: Techniques and Applications. Radiol Res Prac 2014;Article ID 705604, https://doi.org/10.1155/2014/705604

5. Li G, Yang TJ, Furtado H et al. Clinical Assessment of 2D/3D Registration Accuracy in 4 Major Anatomic Sites Using On-Board 2D Kilovoltage Images for 6D Patient Setup. Technol Cancer Res Treat 2015;14(3):305-324

6. Lecchi M, Fossati P, Elisei F et al. Current concepts on imaging in radiotherapy. Eur J Nucl Med Mol Imaging 2008;35(4):821-837

7. Lindfors $\mathrm{N}$, Lund $\mathrm{H}$, Johansson $\mathrm{H}$ et al. Influence of patient position and other inherent factors on image quality in two different cone beam computed tomography (CBCT) devices. Eur J Radiol Open 2017:132-137

8. Keall PJ, Hsu A, Xing L. Image-Guided Adaptive Radiotherapy, Leibel and Phillips Textbook of Radiation Oncology, wyd. Third Edition 2010.

9. Li X. A., Adaptive Radiation Therapy in: Hendee W., Imaging in medical diagnosis and therapy, CRC Press Taylor and Francis Group 2011.

10. Sonke JJ, Aznar M, Rasch C. Adaptive Radiotherapy for Anatomical Changes. Semin Radiat Oncol 2019;29(3):245-257

11. Feldkamp L, Davis L, Kress J. Practical cone-beam algorithm. J Opt Soc Am A 1984;1(6):612-619

12. Srinivasan K, Mohammadi M, Shepherd J. Applications of linac-mounted kilovoltage Cone-beam Computed Tomography on modern radiation therapy: A Review. Pol J Radiol 2014:79:181-93

13. Mao W, Liu C, Gardner SJ et al. Evaluation and Clinical Application of a Commercially Available Iterative Reconstruction Algorithm for CBCT-Based IGRT. Technol Cancer Res Treat 2019:18: 1533033818823054

14. Stock M, Pasler M, Birkfellner W et al. Image quality and stability of image-guided radiotherapy (IGRT) devices: A comparative study. Radiother Oncol 2009;93(1)

15. Moteabbed M, Sharp G, Wang Y et al. Validation of a deformable image registration technique for cone beam CT-based dose verification. Med Phys 2015;42(1):195-205

16. Zoellner C, Rit S, Kurz C et al. Decomposing a prior-CT-based cone-beam CT projection correction algorithm into scatter and beam hardening components. Phys Imag Radiat Oncol 2017;3:49-52

17. Schulze R, Heil U, Gross D et al. Artefacts in CBCT: a review. Dentomaxillofac Radiol 2011;40(5):265-273

18. Kalender WA, Kyriakou Y. Flat-detector Computed Tomography (FD-CT). Eur Radiol 2007;17(11):2767-2779

19. Marchant T, Joshi K, Moore C. Accuracy of radiotherapy dose calculations based on cone-beam CT: comparison of deformable registration and image correction based methods. Phys Med Biol 2018;63(6)

20. Yuan Z, Rong Y, Benedict SH et al. "Dose of the day" based on cone beam computed tomography and deformable image registration for lung cancer radiotherapy. J Appl Clin Med Phys 2020;21(1):88-94

21. Veiga C, McClelland J, Moinuddin S et al. Toward adaptive radiotherapy for head and neck patients: Feasibility study on using CTto-CBCT deformable registration for "dose of the day" calculations. Med Phys 2014;41(3)

22. Oh S, Kim S. Deformable image registration in radiation therapy. Radiat Oncol J 2017;35(2):101-111

23. Rigaud B, Simon A, Castelli $\mathbf{J}$ et al. Deformable image registration for radiation therapy: principle, methods, applications and evaluation. Acta Oncol 2019;58(9):1225-1237

24. Weistrand O, Svensson S. The ANACONDA algorithm for deformable image registration in radiotherapy. Med Phys 2015;42(1):4053

25. Brock KK, Mutic S, McNutt TR et al. Use of image registration and fusion algorithms and techniques in radiotherapy: Report of the AAPM Radiation Therapy Committee Task Group No. 132. Med Phys 2017;44(7)

26. Brock K. K., Hawkins M. A., Eccles C. L. et al., Improving image-guided target localization through deformable registration. Acta Oncol 2008;47(7):1279-1285

27. Thirion J. Image matching as a diffusion process: an analogy with Maxwell's demons. Medical Image Analysis 1998;2(3):243-260

28. Wang H, Dong L, O'Daniel J et al. Validation of an accelerated 'demons' algorithm for deformable image registration in radiation therapy. Phys Med Biol;50(12):2887-2905 
29. Lawson JD, Schreibmann E, Jani AB et al. Quantitative evaluation of a cone-beam computed tomography-planning computed tomography deformable image registration method for adaptive radiation therapy. Journal of Applied Clinical Medical Physics 2007;8(4):96-113

30. Velocity 4.0 Instructions for use. Varian Medical Systems 2018

31. Niu T, Sun M, Star-Lack J et al. Shading correction for on-board cone-beam CT in radiation therapy using planning MDCT images. Med Phys 2010;37(10):5395-5406

32. Kurz C, Dedes G, Resch A et al. Comparing cone-beam CT intensity correction methods for dose recalculation in adaptive intensitymodulated photon and proton therapy for head and neck cancer. Acta Oncol 2015;54(9):1651-1657

33. Laundry G, Dedes G, Zoellner C et al. Phantom Based Evaluation of CT to CBCT Image Registration for Proton Therapy Dose Recalculation. Phys Med Biol 2014;60(2):595-613

34. Kurz C, Kamp F, Park Y-K et al. Investigating deformable image registration and scatter correction for CBCT-based dose calculation in adaptive IMPT. Med Phys 2016;43(10):5635-5646

35. Thing RS, Bernchou U, Mainegra-Hing E et al. Hounsfield unit recovery in clinical cone beam CT images of the thorax acquired for image guided radiation therapy. Phys Med Biol 2016;61(15):5781-5802

36. Thummerer A, Zaffino P, Meijers A et al. Comparison of CBCT based synthetic CT methods suitable for proton dose calculations in adaptive proton therapy. Phys Med Biol 2020;65(9) 